\title{
Increased expression levels of the pvcrt-o and pvmdrl genes in a patient with severe Plasmodium vivax malaria Carmen Fernández-Becerra $^{\dagger 1}$, Maria Jesús Pinazo ${ }^{\dagger 1}$, Ana González${ }^{1}$, Pedro L Alonso ${ }^{1}$, Hernando A del Portillo*1,2 and Joaquim Gascón ${ }^{1}$
}

\author{
Address: ${ }^{1}$ Barcelona Centre for International Health Research (CRESIB), Hospital Clinic/IDIBAPS, Universitat de Barcelona, Roselló 132, 4a \\ planta, 08036, Barcelona, Spain and 2Catalan Institution for Research and Advanced Studies (ICREA), Passeig Lluís Companys, 2308010 \\ Barcelona, Spain \\ Email: Carmen Fernández-Becerra - carmen.fernandez@cresib.cat; Maria Jesús Pinazo - mpinazo@clinic.ub.es; \\ Ana González - portana1@ hotmail.com; Pedro L Alonso - palonso@clinic.ub.es; Hernando A del Portillo* - hernandoa.delportillo@cresib.cat; \\ Joaquim Gascón - JGASCON@clinic.ub.es \\ * Corresponding author †Equal contributors
}

Published: 2 April 2009

Malaria Journal 2009, 8:55 doi:10.1 /86/1475-2875-8-55
Received: 9 December 2008

Accepted: 2 April 2009

This article is available from: http://www.malariajournal.com/content/8/1/55

(c) 2009 Fernández-Becerra et al; licensee BioMed Central Ltd.

This is an Open Access article distributed under the terms of the Creative Commons Attribution License (http://creativecommons.org/licenses/by/2.0), which permits unrestricted use, distribution, and reproduction in any medium, provided the original work is properly cited.

\begin{abstract}
Background: There are increasing reports of severe clinical cases exclusively associated with Plasmodium vivax infections. Notably, this severity has been recently suggested to be associated with chloroquine resistance.

Patients: Two different patients presented at the Hospital Clinic in Barcelona with P. vivax malaria episodes. One patient had severe symptoms and the other mild symptoms. Both patients traveled through the Brazilian Amazon (Manaus) in 2007. For both patients the current diagnosis of malaria was the first. Two other patients with mild symptoms presented to the "Centro de Pesquisa em Medicina Tropical", also in the Brazilian Amazon (Rondônia) in 2000.

Methods: To exclude the possibility that the patient's severe symptoms were due to Plasmodium falciparum, a nested PCR was performed. A magnetic method was used to purify P. vivax free of human leukocytes. Quantitative real-time PCR was performed to compare the transcript levels of two main transporters likely to be involved in chloroquine resistance in $P$. vivax, namely the $P$. vivax chloroquine resistance transporter, pvcrt-o, and the $P$. vivax multidrug resistance transporter, pvmdr $I$.

Results: Results demonstrated that the severe clinical symptoms were exclusively due to $P$. vivax. The patient presented acute respiratory conditions requiring admission to the intensive care unit. The magnetic method showed highly purified infected-reticulocytes with mature stages. In addition, it was found that parasites obtained from the severe patient had up to 2.9-fold increase in pvmdr l levels and up to 21.9-fold increase in pvcrt-o levels compared to expression levels of parasites from the other patients with mild symptoms.

Conclusion: This is the first clinical case of severe disease exclusively associated with vivax malaria in Spain. Moreover, these findings suggest that clinical severity could be associated with increased expression levels of parasite genes likely involved in chloroquine resistance. It is necessary to further explore the potential of pvmdrl and particularly pvcrt-o expression levels as molecular markers of severe disease in $P$. vivax.
\end{abstract}




\section{Background}

The renewed momentum for global malaria eradication has highlighted the need to further studies on Plasmodium vivax if eradication is to be achieved. Although the exact burden of disease is still a matter of debate, it is likely that it has been underestimated and that $P$. vivax is responsible for between 100 and 300 million clinical infections each year [1]. The emergence of worsening clinical severity and chloroquine resistance are two major factors responsible for this increasing burden.

Plasmodium vivax infections have been associated with mild symptoms such as fever, headache, fatigue, chills, and musculoskeletal pain, in particular paroxysms. Recently, however, severe complications, including renal failure, jaundice, acute respiratory distress syndrome, cerebral malaria, seizures, anaemia, hyperparasitaemia, thrombocytopenia, pulmonary edema, splenic rupture and death, have been reported in exclusive association with $P$. vivax $[2,3]$.

Chloroquine resistance (CQR) is a major determinant of the present resurgence of malaria worldwide, including that of P. vivax [4]. Two main transporters have been studied in regard to CQR in P. vivax: the P. vivax chloroquine resistance transporter, Pvcrt-o, and the P. vivax multidrug resistance transporter, Pvmdr1 [5-7]. Interestingly, amino acid polymorphisms have not been associated with chloroquine resistance in pvcrt-o whereas pvmdr1 polymorphisms have been recently suggested to be associated with CQR in Southeast Asia [8]. This data indicates the involvement of other mechanisms in CQR in $P$. vivax. Likely candidates are gene amplifications and differential expression levels $[9,10]$.

The European Network on Imported Infectious Disease Surveillance, TropNetEurop, is an electronic network of clinical sites that monitors imported infectious diseases in Europe http://www.tropnet.net. Since its foundation in 1999, the network has recorded 8,374 cases of malaria, of which close to $11 \%$ (930) were due to $P$. vivax. Worth mentioning, TropNetEurop covers approximately $10 \%$ of all malaria cases reported in Europe. Moreover, according to data from the Spanish National Center of Epidemiology and the Autonomous Government of Catalonia there have been 266 cases of $P$. vivax in Spain in the last six years. In addition, three cases of severe symptoms due to P. vivax in Europe have been reported elsewhere in the literature [11-13]. These figures illustrate the clinical-epidemiological importance of this parasitic disease in a supposedly malaria-free region.

Recently, CQR in P. vivax has been suggested to be associated, albeit not directly linked, with severe vivax malaria [14]. Here, the first clinical case of severe vivax malaria in
Spain is presented. The data also indicates that clinical severity could be associated with increased expression levels of two parasite genes likely involved in chloroquine resistance, pumdr1 and pvcrt-o.

\section{Methods Subjects}

Two patients presented at Hospital Clinic in Barcelona with $P$. vivax malaria episodes. One had severe symptoms and the other mild symptoms. The patients had travelled through the Brazilian Amazon (Manaus) for 31 and 19 days, respectively, in 2007. The patient with severe symptoms was a 30-year-old Spaniard man who had previously travelled to Kenya, in 2006. Upon his return from Brazil, he presented to Hospital Clinic in Barcelona with high fever $\left(39^{\circ} \mathrm{C}\right)$ and a Giemsa-stained thin blood film confirmed the presence of $P$. vivax at a parasitaemia of $1.8 \%$. The patient presented acute respiratory conditions, anaemia and hyperbilirubinaemia, requiring admission to the intensive care unit. The patient with mild symptoms was a 31-year-old Spaniard man who had travelled without chemoprophylaxis and who had previously visited Mexico (2006), Vietnam (2005), and India (2004). There were no records of fever episodes from these previous trips and neither of the patients had ever been diagnosed with malaria.

Two other Brazilian patients with mild symptoms presented at the "Centro de Pesquisa em Medicina Tropical, Rondônia, Brazil in 2000. Total RNA from parasites of these patients was extracted, pooled and stored in liquid nitrogen.

\section{Samples}

Five $\mathrm{mL}$ of infected red blood cells were obtained from each patient. One $\mathrm{mL}$ was used to purify genomic DNA following standard methodologies. The remaining blood was processed to isolate total RNA using the Trizol reagent (Invitrogen) according to the manufacturers' instructions. A recently described magnetic method for the isolation of matures stages of malaria parasites was used to concentrate and purify $P$. vivax-infected reticulocytes [15]. Giemsa-stained smears showed an absence of human leukocytes, and all the reticulocytes were infected with mature stages of the parasite (Additional file 1). Eluents were centrifuged at $800 \times \mathrm{g}$ for 10 minutes, supernatants discarded, and pellets used to purify total RNA. The protocol for this study was approved by the Ethical Committee of Hospital Clinic and informed consent obtained from the patients.

\section{Nested PCR}

Nested polymerase chain reaction (PCR) was performed as previously described [16] to exclude $P$. falciparum infec- 
tions. Fragments were resolved and visualized on 2\% agarose gels stained with sybr green.

\section{qRT-PCR}

Amplification reactions were performed using Power SYBR Green PCR Master Mix (Applied Biosystems) and 45 ng of template cDNA prepared from each sample. Samples were set up in duplicate and experiments were repeated independently twice. PCR products were amplified and detected on an ABI Prism 7700 (Applied Biosystems). Cycling parameters for PCR were an initial denaturation step at $95^{\circ} \mathrm{C}$ for 10 minutes, followed by 40 cycles of $95^{\circ} \mathrm{C}$ for 15 seconds, and $60^{\circ} \mathrm{C}$ for 1 minute. To analyse the relative transcript levels, the threshold cycle value $(\mathrm{Ct})$ of each sample was used to calculate and compare the $\Delta \mathrm{Ct}$ of each sample to that of the P. vivax housekeeping gene Sal I $\beta$-tubulin; the $\Delta \Delta \mathrm{Ct}$ was also calculated as in [17] to compare the transcript levels of pvcrt-o and pumdr 1 in the patient with severe symptoms and in the patient with mild symptoms.

\section{Results}

\section{Case report}

During the month of August, 2007, a 30-year-old Spanish tourist traveled through the Brazilian Amazon region of Manaus, where, due to gastric disturbances, he took an incomplete chemoprophylaxis consisting of proguanil and chloroquine. Upon his return to Spain 30 days later, he presented to a health center with an eight-day history of fever, chills and dry cough. He was diagnosed with lower respiratory tract infection and treated with amoxicillin-clavulanic acid 875/125 mg every 8 hours for 24 hours ( 3 doses) without resolution. On presenting a week later to Hospital Clinic in Barcelona, he had high fever $\left(39^{\circ} \mathrm{C}\right)$ and jaundice; the tip of the spleen was palpable and chest auscultation was unremarkable. A thin blood smear of peripheral blood stained with Giemsa revealed $P$. vivax infection with a parasitaemia of $1.8 \%$. The patient presented acute respiratory conditions requiring admission to the intensive care unit (ICU).

In the ICU, he was haemodynamically stable and blood tests revealed pancytopaenia (haemoglobin, $10 \mathrm{~g} / \mathrm{L}$; haematocrit, 29\%; platelets $25 \times 10^{9} / \mathrm{L}$, leukocytes, $6.7 \times 10^{9} /$ L); hyperbilirubinaemia $(8.3 \mathrm{~g} / \mathrm{dL}) ; \gamma$-glutamyltransferase, $146 \mathrm{U} / \mathrm{L}$; alkaline phosphatase, $241 \mathrm{U} / \mathrm{L}$; and prothrombin time, 76 seconds. Renal function tests were within normal limits. The C-reactive protein level was 16 $\mathrm{mg} / \mathrm{dL}$. Arterial blood gas measurement while breathing air revealed marked hypoxia ( $\mathrm{PaO} 2,63 \mathrm{~mm} \mathrm{Hg})$, normocapnia (PaCO2, $32 \mathrm{~mm} \mathrm{Hg}$ ), low oxygen saturation $(93.7 \%)$, and a blood $\mathrm{pH}$ of 7.49 . A chest radiograph showed bilateral interstitial infiltrates and a computed tomography (CT) scan showed right midzone alveolar shadowing without parenchymal infiltrations (Figures 1A and $1 \mathrm{~B})$.

To exclude the possibility that the patient's severe symptoms were due to $P$. falciparum, which is sympatric with $P$. vivax in Brazil, a nested PCR using $P$. falciparum- and $P$. vivax-specific primers, was performed [16]. The results demonstrated that coinfection with $P$. falciparum could be excluded (Figure 2A). Moreover, sputum, blood, and nasopharyngeal swab samples obtained for culture were negative, as were serological tests for atypical respiratory pathogens, human immunodeficiency virus, histoplasma, coccidians, and paracoccidians. Together, these results demonstrated that the clinical symptoms were exclusively due to $P$. vivax infection. a

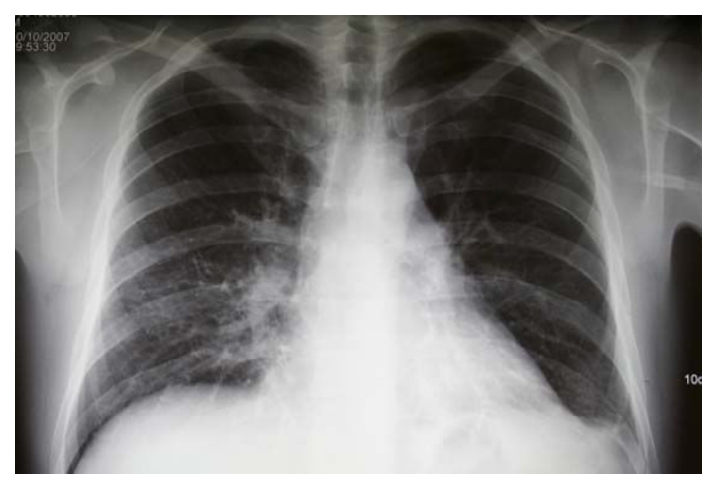

b

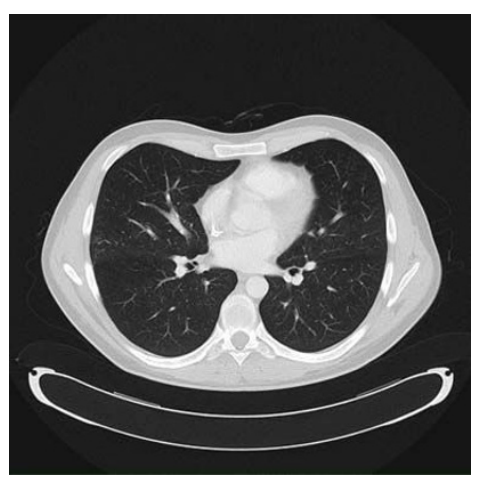

C

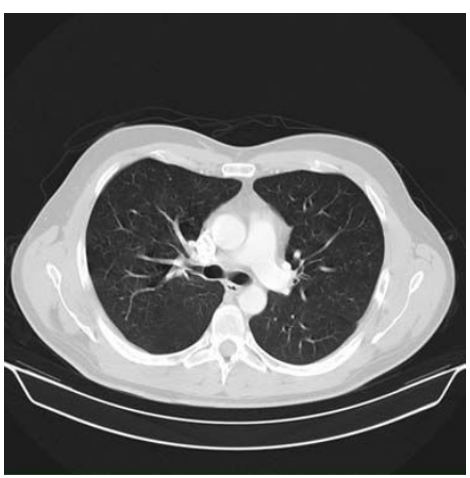

Figure I

X-ray and computed tomography (CT) scans of patient with severe Plasmodium vivax malaria. Interstitial bilateral infiltrates compatible with acute lung injury in X-ray (Panel A). Infiltrates in right midzone in CT scan (panel B). Normal CT scan two months after acute episode (panel C). 

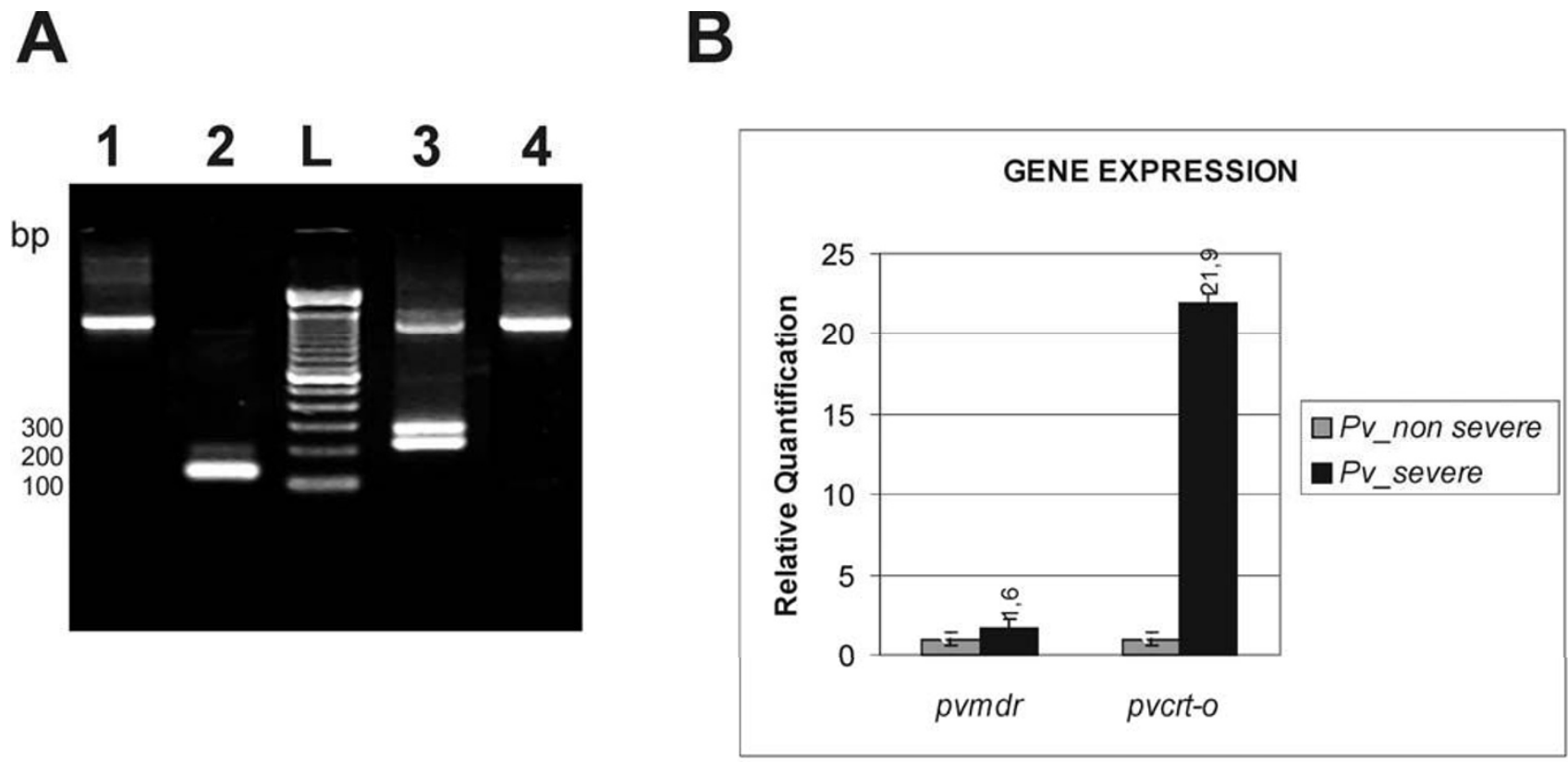

Figure 2

Expression levels of chloroquine resistance genes in severe and mild Plasmodium vivax malaria. In panel $A$, nested PCR to identify Plasmodium species. Amplification of gDNA from $P$. falciparum 3D7 strain and $P$. vivax from severe patient, respectively, using specific $P$. vivax primers (lanes I and 2). Amplification of gDNA from $P$. falciparum 3D7 strain and $P$. vivax from severe patient, respectively, using specific $P$. falciparum primers (lanes 3 and 4). Molecular weight ladder (lane $L$ ). A positive reaction is noted when primers for $P$. falciparum and $P$. vivax produce amplification products of 205-bp and $120-b p$, respectively [16]. Molecular weight in base-pairs (bp). In panel B, relative quantification of pvcrt-o and pvmdrl transcripts in total RNA obtained from parasites from the severe patient vs total RNA obtained from parasites from a patient from Brazil with $P$. vivax and non-severe symptoms also presenting to our hospital. The following oligonucleotide primers were designed for the realtime experiments using the Primer Express program (Applied Biosystems). Primers: F-pvcrt-oRT 5'-ATGTCCAAGATGTGCGACGAT-3';R-pvcrt-oRT 5'-CTGGTCCCTGTATGCAACTGAC-3'; F-pvmdr/RT 5'-AAGGATCAAAGGCAACCCA-3'; RpvmdrIRT5'-TCAGGTTGTTACTGCTGTTGCTATT-3'; F-pvtubulinRT 5' CCAAGAATATGATGTGTGCAAGTG 3'; R-pvtubulinRT 5' GGCGCAGGCGGTTAGG 3'.

Due to the severe condition of the patient, a five-day treatment was initiated, starting with an intravenous loading dose of quinine $(1,200 \mathrm{mg})$ on the first day, followed by oral quinine $(600 \mathrm{mg}$ ) every 8 hours plus oral doxycycline (100 mg) every 12 hours. The treatment was well-tolerated and parasitaemia became negative within three days. Platelet, erythrocyte and leukocyte levels were all within normal ranges in the following controls. Normal blood levels of glucose-6-phosphate dehydrogenase activity were detected and oral primaquine (30 mg/day) was commenced for two weeks to prevent a relapse. The patient recovered well and the chest $\mathrm{CT}$ findings were normal at two months (Figure 1C). There have been no recurrences during the follow-up period.

\section{Increased expression levels of drug resistance genes}

In view of the recently suggested association of severe disease in $P$. vivax with multidrug resistance [14], expression levels of two genes suspected in having a pivotal role in mediating clinical resistance to chloroquine, pvcrt-o and pvmdr1, were determined. Significantly, the patient with severe symptoms had a 1.6-fold increase in pumdr1 levels and a 21.9-fold increase in pvcrt-o levels compared to the other patient who presented to the same hospital with $P$. vivax infection and mild symptoms (Figure $2 \mathrm{~b}$ ). This finding was validated by analysing parasite material obtained previously from two other patients from the Brazilian Amazon who also had mild symptoms (Additional file 2).

\section{Discussion}

There are increasing reports of severe clinical cases exclusively associated with $P$. vivax infections, involving severe anaemia, renal failure, jaundice, cerebral malaria, seizures, respiratory failure, multi-organ failure, and death $[2,3]$; all these complications are generally believed to be exclusively associated with severe forms of falciparum malaria. Although these same clinical severity criteria are being used for $P$. vivax, it would be desirable to conduct prospective studies to establish a precise definition of clinical severity in $P$. vivax. 
The patient with severe symptoms had acute lung injury according to the definition of the American-European Consensus Conference on ARDS [18], with acute onset, bilateral changes in chest radiography, a $\mathrm{PaO} 2 / \mathrm{FiO} 2$ ratio of $\leq 300$, and absence of clinical left ventricular failure. Importantly, these respiratory complications appeared before initiation of anti-malarial drug treatment, an observation also reported in two other severe clinical cases of lung injury due to $P$. vivax $[19,20]$. Two main hypotheses have been proposed to explain lung damage in $P$. vivax malaria infections. The first suggests that there is no sequestration of $P$. vivax-infected reticulocytes in the deep capillaries of internal organs, postulating instead an inflammatory process due to an increase in capillary permeability associated with cytokine-induced damage in the pulmonary epithelium [21]. The second suggests cytoadherence of $P$. vivax-infected reticulocytes in lung capillaries, causing obstruction of blood flow and reduction of respiratory function before treatment and alveolar capillary damage and inflammation 24 to 48 hours after initiation of anti-malarial drug treatment [22]. The possibility that antibiotics given 24 hours before respiratory failure might have destroyed P. vivax-infected reticulocytes, inducing lung damage and favouring the second hypothesis, cannot formally rule out. Yet, it is clear that the lack of sequestration in P. vivax needs to be re-evaluated, as cytoadherence has been hypothesized to occur in both the spleen $[23,24]$ and the lungs [22].

Chloroquine is currently the first-line treatment for $P$. vivax, but resistance has been rapidly increasing since it was first described in two cases of treatment failures in Papua New Guinea [4]. The clinical case reported here originated in Manaus, Brazil, where P. vivax chloroquine resistance and severe disease are now being reported $[25,26]$. Interestingly, after the appearance of chloroquine resistance in $P$. vivax, reports of clinical severity exclusively associated with this human malaria parasite started to appear [2]. Moreover, multidrug resistance has been recently suggested to be associated, albeit not directly linked, with severe disease in P. vivax [14]. Remarkably, higher expression levels of pumdr1 and pvcrt-o, in particular, were found in parasites from the patient with severe clinical symptoms compared to three patients with mild symptoms. The levels were increased by up to 2.9 fold in the case of pumdr1 (when including all samples) and up to 21.9 fold in the case of pvcrt-o. Confounding effects due to sample concentrations or contamination with $P$. falciparum or human material were excluded by using $P$. vivaxspecific primers and $\beta$-tubulin as an internal control and calibrator. This data thus indicates that severe vivax disease could be associated to molecular markers.

\section{Conclusion}

There are increasing reports of severe disease exclusively associated with vivax malaria and this study presents the first one in Spain. The use of PCR excluded incompetent microscopy, cryptic mixed infections or sequestered $P$. falciparum reinforcing the need of using PCR as a new diagnostic tool to avoid default diagnosis of severe malaria as due to $P$. falciparum. The finding on increased expression levels of parasite genes likely involved in chloroquine resistance supports to furthering explore the potential of pvmdr1 and particularly pvcrt-o as molecular markers of severe disease in P. vivax.

\section{Competing interests}

The authors declare that they have no competing interests.

\section{Authors' contributions}

All clinical aspects of these patients were studied by MP, $\mathrm{AG}$, and JG.

All molecular data on DNA and RNA were produced and analysed by CFB and HdP.

PA critically reviewed the manuscript

CFB, MP, JG and HdP drafted the manuscript

\section{Additional material}

\section{Additional file 1}

Plasmodium vivax purification with MACS. Giemsa-stained thin smears representing mature forms of Plasmodium vivax free of human leukocytes after purification using MACS.

Click here for file

[http://www.biomedcentral.com/content/supplementary/1475-

2875-8-55-S1.pdf]

\section{Additional file 2}

Expression levels of chloroquine resistance genes in severe and mild Plasmodium vivax malaria. Relative quantification of pvcrt-o and pvmdr1 transcripts in total RNA obtained from parasites of severe and non-severe P. vivax patients.

Click here for file

[http://www.biomedcentral.com/content/supplementary/1475-

2875-8-55-S2.pdf]

\section{Acknowledgements}

We are grateful to Neus Cardeñosa (Dirección General de Salud Pública, Departamento de Salud, Generalitat de Catalunya) and Luisa Pilar Sánchez (Centro Nacional de Epidemiología) for providing us with data on malaria cases in Catalonia and Spain. Work in the laboratory of HAP is funded by the Fundación Privada CELLEX (Catalonia, Spain). 


\section{References}

I. Price RN, Tjitra E, Guerra CA, Yeung S, White NJ, Anstey NM: Vivax malaria: neglected and not benign. Am J Trop Med Hyg 2007, 77(6 Suppl):79-87.

2. Baird JK: Neglect of Plasmodium vivax malaria. Trends Parasitol 2007, 23:533-539.

3. Kochar DK, Das A, Kochar SK, Saxena V, Sirohi P, Garg S, Kochar A, Khatri MP, Gupta V: Severe Plasmodium vivax malaria: a report on serial cases from Bikaner in northwestern India. Am J Trop Med Hyg 2009, 80:194-198.

4. Baird JK: Chloroquine resistance in Plasmodium vivax. Antimicrob Agents Chemother 2004, 48:4075-4083.

5. Nomura T, Carlton JM, Baird JK, del Portillo HA, Fryauff DJ, Rathore D, Fidock DA, Su X, Collins WE, McCutchan TF, Wootton JC, Wellems TE: Evidence for different mechanisms of chloroquine resistance in 2 Plasmodium species that cause human malaria. J Infect Dis 200I, I83:1653-166I

6. Brega S, Meslin B, de Monbrison F, Severini C, Gradoni L, Udomsangpetch R, Sutanto I, Peyron F, Picot S: Identification of the Plasmodium vivax mdr-like gene (pvmdrl) and analysis of singlenucleotide polymorphisms among isolates from different areas of endemicity. J Infect Dis 2005, I 91:272-277.

7. Sa JM, Nomura T, Neves J, Baird JK, Wellems TE, del Portillo HA: Plasmodium vivax: allele variants of the mdrl gene do not associate with chloroquine resistance among isolates from Brazil, Papua, and monkey-adapted strains. Exp Parasitol 2005, I 09:256-259.

8. Suwanarusk R, Russell B, Chavchich M, Chalfein F, Kenangalem E, Kosaisavee V, Prasetyorini B, Piera KA, Barends M, Brockman A, Lek Uthai U, Anstey NM, Tjitra E, Nosten F, Chen Q, Price RN: Chloroquine resistant Plasmodium vivax: in vitro characterisation and association with molecular polymorphisms. PLOS ONE 2007, 2:e 1089

9. Imwong M, Pukrittayakamee S, Pongtavornpinyo W, Nakeesathit S, Nair S, Newton P, Nosten F, Anderson TJ, Dondorp A, Day NP, White NJ: Gene amplification of Plasmodium vivax multidrug resistance I gene in Thailand, Laos, and Myanmar. Antimicrob Agents Chemother 2008, 52:2657-2659.

10. Sa JM, Yamamoto MM, Fernandez-Becerra C, de Azevedo MF, Papakrivos J, Naude B, Wellems TE, Del Portillo HA: Expression and function of pvcrt-o, a Plasmodium vivax ortholog of pfcrt, in Plasmodium falciparum and Dictyostelium discoideum. Mol Biochem Parasitol 2006, I 50:21 9-228.

II. Kumar S, Melzer M, Dodds P, Watson J, Ord R: P. vivax malaria complicated by shock and ARDS. Scand J Infect Dis 2007, 39:255-256.

12. Lawn SD, Krishna S, Jarvis JN, Joet T, Macallan DC: Case reports: pernicious complications of benign tertian malaria. Trans $R$ Soc Trop Med Hy 2003, 97(5):55I-553.

13. Tanios MA, Kogelman L, McGovern B, Hassoun PM: Acute respiratory distress syndrome complicating Plasmodium vivax malaria. Crit Care Med 200I, 29:665-667.

14. Tjitra E, Anstey NM, Sugiarto P, Warikar N, Kenangalem E, Karyana M, Lampah DA, Price RN: Multidrug-resistant Plasmodium vivax associated with severe and fatal malaria: a prospective study in Papua, Indonesia. PLoS Medicine 2008, 5: el 28.

15. Trang DT, Huy NT, Kariu T, Tajima K, Kamei K: One-step concentration of malarial parasite-infected red blood cells and removal of contaminating white blood cells. Malar J 2004, 3:7.

16. Snounou G, Singh B: Nested PCR analysis of Plasmodium parasites. Methods Mol Med 2002, 72:189-203.

17. Livak KJ, Schmittgen TD: Analysis of relative gene expression data using real-time quantitative PCR and the 2(-Delta Delta C(T)) Method. Methods 200I, 25:402-408.

18. Bernard GR, Artigas A, Brigham KL, Carlet J, Falke K, Hudson L, Lamy M, Legall JR, Morris A, Spragg R: The American-European Consensus Conference on ARDS. Definitions, mechanisms, relevant outcomes, and clinical trial coordination. Am J Resp Crit Care Med 1994, 149:818-824.

19. Lomar AV, Vidal JE, Lomar FP, Barbas CV, de Matos GJ, Boulos M: Acute respiratory distress syndrome due to vivax malaria: case report and literature review. Braz J Infect Dis 2005, 9:425-430.

20. Munteis E, Mellibovsky L, Marquez MA, Minguez S, Vazquez E, Diez A: Pulmonary involvement in a case of Plasmodium vivax malaria. Chest 1997, I I I:834-835.
21. Price L, Planche T, Rayner C, Krishna S: Acute respiratory distress syndrome in Plasmodium vivax malaria: case report and review of the literature. Trans $R$ Soc Trop Med Hyg 2007, I $01: 655-659$.

22. Anstey NM, Handojo T, Pain MC, Kenangalem E, Tjitra E, Price RN, Maguire GP: Lung injury in vivax malaria: pathophysiological evidence for pulmonary vascular sequestration and posttreatment alveolar-capillary inflammation. J Infect Dis 2007, 195:589-596.

23. Fernandez-Becerra C, Yamamoto MM, Vencio RZ, Lacerda $M$, Rosanas-Urgell A, Del Portillo HA: Plasmodium vivax and the importance of the subtelomeric multigene vir superfamily. Trends Parasitol 2009, 25:44-5I.

24. del Portillo HA, Lanzer M, Rodriguez-Malaga S, Zavala F, FernandezBecerra $C$ : Variant genes and the spleen in Plasmodium vivax malaria. Int J Parasit 2004, 34: I 547-I 554.

25. de Santana Filho FS, Arcanjo AR, Chehuan YM, Costa MR, MartinezEspinosa FE, Vieira JL, Barbosa MG, Alecrim WD, Alecrim MG: Chloroquine-resistant Plasmodium vivax, Brazilian Amazon. Emerging Infect Dis 2007, I 3: I I 25- I I 26.

26. Lacerda MV, Alexandre MA, Santos PD, Arcanjo AR, Alecrim WD, Alecrim MG: Idiopathic thrombocytopenic purpura due to vivax malaria in the Brazilian Amazon. Acta Trop 2004, 90:187-190.
Publish with Biomed Central and every scientist can read your work free of charge

"BioMed Central will be the most significant development for disseminating the results of biomedical research in our lifetime. "

Sir Paul Nurse, Cancer Research UK

Your research papers will be:

- available free of charge to the entire biomedical community

- peer reviewed and published immediately upon acceptance

- cited in PubMed and archived on PubMed Central

- yours - you keep the copyright

Submit your manuscript here:

http://www.biomedcentral.com/info/publishing_adv.asp
BioMedcentral 